\title{
Oficinas de Atividades: Reconstruindo o Cotidiano de Pacientes Submetidos ao Transplante de Medula Óssea
}

\author{
Workshops of Activities: Rebuilding the Daily Life of the \\ Patients Undergoing Bone Marrow Transplantation
}

\section{RESUMO}

O Transplante de Medula Óssea (TMO) é um procedimento de alta complexidade, cujo desenvolvimento permitiu o tratamento de doenças que, anteriormente, eram fatais. Como parte do processo de reabilitação, foram implementadas oficinas terapêuticas com a finalidade primordial de estimular a socialização e a troca de experiências entre pacientes e acompanhantes. Além desses propósitos, as oficinas funcionavam como um espaço de aprendizado para profissionais em formação. O objetivo deste estudo é descrever o funcionamento das oficinas terapêuticas oferecidas na sede do Grupo de Apoio ao Transplantado de Medula Óssea (GATMO) e investigar as percepções de seus participantes em relação às atividades desenvolvidas. As oficinas terapêuticas do GATMO foram realizadas semanalmente, coordenadas por estagiários-bolsistas do curso de Psicologia da Faculdade de Filosofia, Ciências e Letras de Ribeirão Preto da Universidade de São Paulo (FFCLRP-USP) e frequentadas por pacientes e seus acompanhantes (mínimo de quatro, máximo de 12 pessoas). Os resultados obtidos evidenciam que as oficinas se configuraram como meio de expressão de sentimentos, ferramenta de resgate da capacidade produtiva e de socialização. Observou-se que as produções dos usuários, como artesanatos e mosaicos, extrapolam os limites dos workshops, uma vez que as tarefas também são realizadas em outros contextos, permitindo que o paciente desempenhe atividades alternativas, de modo a ampliar seu repertório ocupacional.

Palaviras-chave: Transplante de Medula Óssea. Oficina Terapêutica. Psicologia. Cuidadores. Grupo.

\section{ABSTRACT}

Bone Marrow Transplantation (BMT) is a procedure of high complexity, whose development has allowed the treatment of diseases that were previously fatal. As part of the rehabilitation process, workshops were implemented, with the primary purpose to stimulate socialization and exchange of experiences among patients and caregivers.

Flávia Andréa Prado

Patrocínio, érica

Arantes de oliveira-

Cardoso, Bruna

Vieira Von Zuben e

Manoel Antônio Dos

Santos

Universidade de São Paulo. Faculdade de Filosofia, Ciências e Letras de Ribeirão Preto, São Paulo, Brasil 
In addition, the workshops functioned as a learning space for trainees. The aim of this study is to describe the functioning of the therapeutic workshops offered at head office of the Support Group for Bone Marrow Transplanted (GATMO, in portuguese) and investigate the perception of participants in relation to the activities developed. Therapeutic workshops were held weekly, coordinated by interns and fellows of psychology and attended by patients and their caregivers (minimum four, maximum of 12 people). The results obtained show that the workshops are characterized as spaces for expression of feelings, recovery of productive capacity and socialization. It was observed that the productions of users extrapolate the limits of workshops, since the tasks are also conducted in other contexts, allowing the patient to perform alternative activities in order to broaden his/her occupational repertoire.

Keywords: Bone Marrow Transplantation. Therapeutic Workshops. Psychology. Caregivers. Group.

\section{INTRODUÇÃO}

Atualmente, o Transplante de Medula Óssea (TMO) é o recurso mais adequado e mais suscetível ao sucesso no tratamento de diversas doenças graves, outrora invariavelmente fatais, como a leucemia e outras neoplasias. Trata-se, no entanto, de um procedimento médico complexo, extenso e agressivo, passível de intercorrências adversas, com consequências imprevisíveis e potencialmente fatais. Experiências dolorosas, de ordem física, social e psicológica, são vivenciadas pelos pacientes submetidos a esse tratamento [6].

Quadros clínicos de depressão, ansiedade, descontrole, perda da motivação e da orientação, angústia e medo da morte, são estados bastante frequentes. Além disso, o paciente padece de dores e pode enfrentar complicações decorrentes do tratamento. A radioterapia e a quimioterapia acarretam efeitos colaterais significativos e debilitantes. Complicações decorrentes de quadros infecciosos são possíveis e não muito raras. Esses pacientes têm que aprender a lidar com o isolamento, que implica ruptura com o cotidiano, separação do local de moradia e dos familiares, convivendo com a constante companhia da angústia da possibilidade de morte iminente. Concomitantemente, a família também é acometida por muitos sentimentos negativos, agravados com o estresse produzido pela ruptura do cotidiano, alterações nas funções e papéis familiares, despesas elevadas e incertezas em relação ao futuro [2].

Os pacientes têm seu sofrimento intensificado especialmente pelo fato de que, ao chegarem à unidade de TMO, já passaram por diversos serviços e se submeteram a uma rotina desgastante de exames, tratamentos, consultas e retornos médicos, na busca de dispositivos de saúde que, enfim, possam oferecer reais possibilidades de cura. Muitas vezes observa-se, nessa situação, um latente sofrimento por antecedência. Durante o tratamento, o peso das rotinas rígidas estabelecidas por protocolos, o tempo prolongado de confinamento e a necessidade de isolamento em um sistema diferenciado de internação colaboram com o agravamento desse sofrimento, sem contar as constantes previsões acerca de reações e efeitos colaterais que podem vir 
a ocorrer [6]. As pressões físicas e psicológicas que os pacientes se veem obrigados a suportar colocam toda sua trajetória de vida em perspectiva. Por outro lado, ao estresse e sofrimento persistentes se contrapõe a promessa de que tudo pode melhorar a partir do tratamento.

O reconhecimento da interação existente entre aspectos físicos e psicológicos no correr de um quadro grave de adoecimento, e no enfrentamento do tratamento específico para tal quadro, resultou na inclusão do psicólogo nas equipes de cuidado multidisciplinar, especialmente na área da oncologia e onco-hematologia. Essa inclusão é relativamente recente nos contextos de tratamento. Na Unidade de TMO do Hospital das Clínicas da Faculdade de Medicina de Ribeirão Preto (HC-FMRP-USP), a intervenção psicológica inicia-se antes mesmo do início do procedimento (etapa pré-TMO), atravessa o período de hospitalização, durante o qual o procedimento é realizado (etapa do TMO propriamente dita), além de contribuir com a reabilitação psicossocial do paciente após o procedimento (etapa pós-TMO). A intervenção psicológica tem se afigurado como um recurso de importância crucial, na medida em que amplia os limites da ação da equipe médica no tocante a acolher as demandas de saúde mental que acompanham a trajetória do transplante e que impactam tanto os pacientes como seus familiares em cada etapa do tratamento [2].

As técnicas de grupo se apresentam como recurso auxiliar interessante, pois colaboram com o oferecimento de assistência a um número mais elevado de pessoas. Além disso, permitem adequações a diversas situações e contextos, especialmente as técnicas que abrem brechas para a vivência do lúdico, outro importante elemento que facilita a expressão de sentimentos e anseios reprimidos, sobretudo em relação ao medo da morte ou da separação, às incertezas quanto ao futuro e ao novo modo de vida a ser adotado [1].

Oliveira-Cardoso et al. [5] investigaram a qualidade de vida de 17 pacientes submetidos ao TMO em três momentos distintos: pré-TMO, no momento da saída da enfermaria e após um ano do procedimento. Os resultados mostraram que, no momento da alta hospitalar, há um decréscimo significativo da qualidade de vida quando comparada com o período anterior, merecendo destaque os prejuízos nos aspectos sociais (avalia a frequência da interferência nas atividades sociais devido a problemas físicos ou emocionais) e físicos (avaliam se as limitações físicas e quanto essas dificultam a realização de trabalho e atividades diárias).

Outro estudo realizado no mesmo serviço, com objetivo de verificar a relação entre renda, trabalho e qualidade de vida em 62 pacientes transplantados de medula óssea que permaneciam em acompanhamento médico, proporcionou um entendimento mais claro da capacidade laboral desses indivíduos nessa fase. Observou-se que $66,10 \%$ dos sujeitos da amostra não exerciam qualquer atividade remunerada, sendo que uma parcela significativa dos pacientes que haviam retomado o trabalho teve que mudar suas ocupações anteriores, uma vez que o esforço físico de alta intensidade e a exposição prolongada ao sol são aspectos contraindicados para transplantados, o que limita o repertório ocupacional e as possibilidades de readaptação funcional. Além disso, pacientes com maior renda familiar apresentavam melhor qualidade de vida [3].

O Grupo de Apoio ao Transplantado de Medula Óssea (GATMO) mantém, no 
campus de Ribeirão Preto-SP da Universidade de São Paulo, uma casa que hospeda pacientes e acompanhantes de outras cidades e estados do país, com parcos recursos socioeconômicos, durante os períodos pré e pós-transplante. Essa casa de apoio é importante porque permite manter os pacientes próximos ao HC-FMRP-USP, facilitando seu acesso ao acompanhamento diário na Unidade de TMO, no período imediatamente após a alta da enfermaria.

Considerando as características dessa clientela, mostrou-se necessário implementar atividades de reabilitação, na forma de workshops terapêuticos, com a finalidade de estimular a capacidade produtiva, socialização, expressão e troca de experiências entre os pacientes e os acompanhantes, além de funcionar como um espaço de aprendizado para profissionais em formação.

O objetivo deste estudo é descrever o funcionamento das oficinas terapêuticas oferecidas na casa de apoio do GATMO e investigar as percepções de seus participantes em relação às atividades desenvolvidas.

\section{MATERIAIS E MÉTODOS}

Este estudo caracteriza-se por ser descritivo e exploratório, de abordagem qualitativa, desenvolvido junto a uma casa de apoio como parte de um projeto de extensão universitária.

As oficinas terapêuticas do GATMO foram realizadas semanalmente, com duração de duas horas, coordenadas por estagiários e bolsistas do curso de Psicologia da FFCLRP-USP e frequentadas por pacientes transplantados e seus acompanhantes. As atividades foram estruturadas em função dos objetivos descritos, abrangendo quatro categorias: dinâmicas de grupo, artesanato, jogos e apresentação seguida de discussão de filmes.

Durante o período de $1^{\mathrm{o}}$ de agosto de 2012 a 31 de julho de 2013, foram realizadas 43 oficinas, atendendo um total de 205 pessoas, entre pacientes e acompanhantes. As percepções dos participantes em relação às tarefas desenvolvidas foram coletadas no transcurso das atividades ou posteriormente, em diálogos estabelecidos entre os participantes e as coordenadoras do grupo.

As falas foram registradas por uma das coordenadoras, concomitantemente à realização das atividades, compondo o corpus do estudo, juntamente com as fotografias das produções realizadas em cada oficina. $\mathrm{O}$ material verbal e pictórico foi analisado segundo os procedimentos de análise de conteúdo, que permitiram elencar as seguintes categorias temáticas:

1. Grupo como meio de expressão de sentimentos;

2. Grupo como ferramenta de resgate da produtividade;

3. Grupo como espaço de socialização. 


\section{RESULTADOS E DISCUSSÃO}

As oficinas foram frequentadas por, no mínimo, quatro e, no máximo, 12 pessoas.

\section{Grupo como meio de expressão de sentimentos}

O grupo se configurou como um espaço privilegiado para a expressão dos sentimentos, de forma verbal ou por meio de ações e envolvimento com as atividades propostas, conforme evidencia a fala do paciente:

Pra mim o grupo é muito importante porque é através das atividades que fazemos juntos que eu posso expressar coisas que eu não conseguiria mostrar se não fosse dessa forma. ( $M, 21$ anos, masculino, diagnóstico de leucemia).

No espaço grupal, os participantes falavam e retratavam a saudade de casa, mencionavam a falta que sentiam da rotina que tinham antes do adoecimento, a necessidade de seguir as imposições e se ajustar às limitações impostas pelo tratamento e o medo da recidiva da doença. Porém, também trocavam experiências, se apoiavam mutuamente e se ajudavam a superar as barreiras que encontravam pelo caminho.

A gente fica mais próximo, né? Vai fazendo e nem vê o tempo passar. Vai conversando e conhecendo os outros. Ajuda a falar das tristezas e das esperanças, e saber que tem estas pessoas que vêm só para ajudar a gente, né? (J, 42 anos, feminino, diagnóstico de leucemia).

Um recurso bastante utilizado pelas crianças e adolescentes transplantados de medula óssea foram as pinturas e desenhos. Durante e após essas atividades, os coordenadores estimulavam uma conversa sobre o conteúdo dos desenhos e sobre o que essas produções poderiam estar ilustrando ou representando naquele momento para o paciente. A Figura 1 mostra uma foto de algumas das telas pintadas e que animaram as conversações.

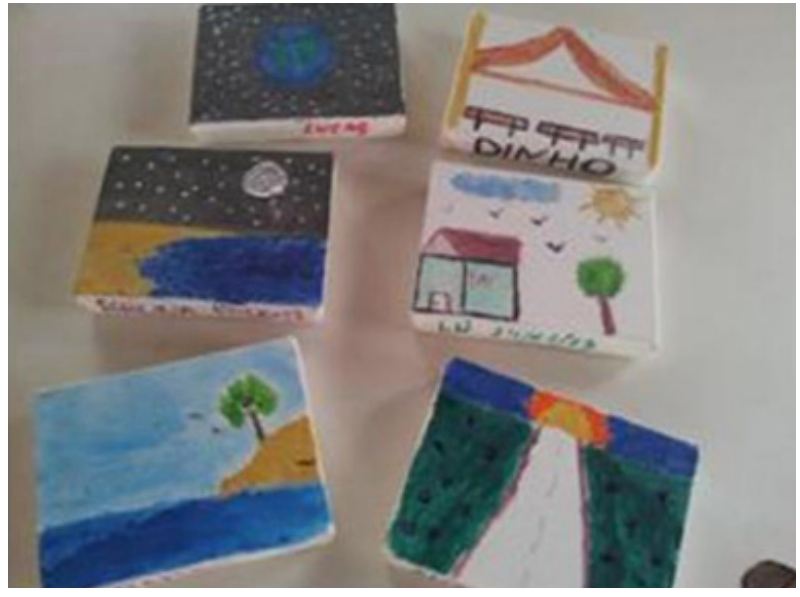

Figura 1 - Pinturas realizadas por crianças e adolescentes durante as oficinas do GATMO 
Apesar de não ter a proposta de profissionalização, as oficinas auxiliavam no resgate da capacidade produtiva, tão comprometida durante o tratamento e bastante restrita imediatamente à alta da enfermaria.

Não estou mais no GATMO, graças a Deus já estou em casa, mas sempre que volto nos retornos dou uma passada no grupo. Acho que foi importante ter esse lugar durante o meu tratamento, para poder pensar que eu poderia fazer alguma coisa, que nem tudo era "não". ( $M, 36$ anos, feminino, diagnóstico de Leucemia Mielóide Crônica).

Alguns pacientes, inclusive, relataram que expandiram essa experiência para além do espaço grupal, fazendo as atividades na Casa do GATMO fora dos horários dos grupos, ou mesmo em casa, após a alta ambulatorial.

Figura 2 - Mosaicos produzidos durante as oficinas do GATMO

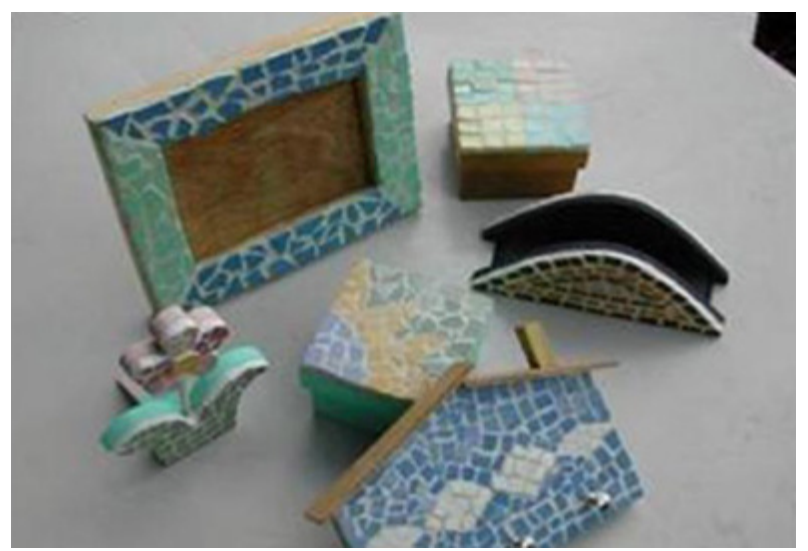

Cheguei meio perdido no GATMO, mas percebi que tinha um grupo de pacientes que esperava o horário dessa tal oficina para aprender coisas. Não sabia bem o que era, mas fui atrás deles. Hoje faço os melhores mosaicos e pego até encomendas. (F, 18 anos, masculino, diagnóstico de leucemia).

\section{Grupo como espaço de socialização}

A possibilidade de engajar-se em um encontro semanal, no qual o foco fosse retirado da doença e do tratamento e colocado na qualidade de vida e no treinamento de habilidades que estimulavam a imaginação criativa, aparece como principal contribuição das oficinas.

Assim que eu cheguei ao GATMO me falaram do pessoal que vinha fazer atividades na sexta à tarde e de como era bom. A gente passa a semana inteira na Casa, naquela rotina, cuidando do paciente, que nem sobra tempo pra mais nada. Muitas vezes nós, acompanhantes, nem conseguimos tempo para falar com os outros. Esse espaço é importante porque a gente consegue parar e falar do que está passando, ouvir 
o outro, mas também de outras coisas, às vezes até falamos do futuro. (F, 35 anos, mãe de um adolescente com diagnóstico de leucemia).

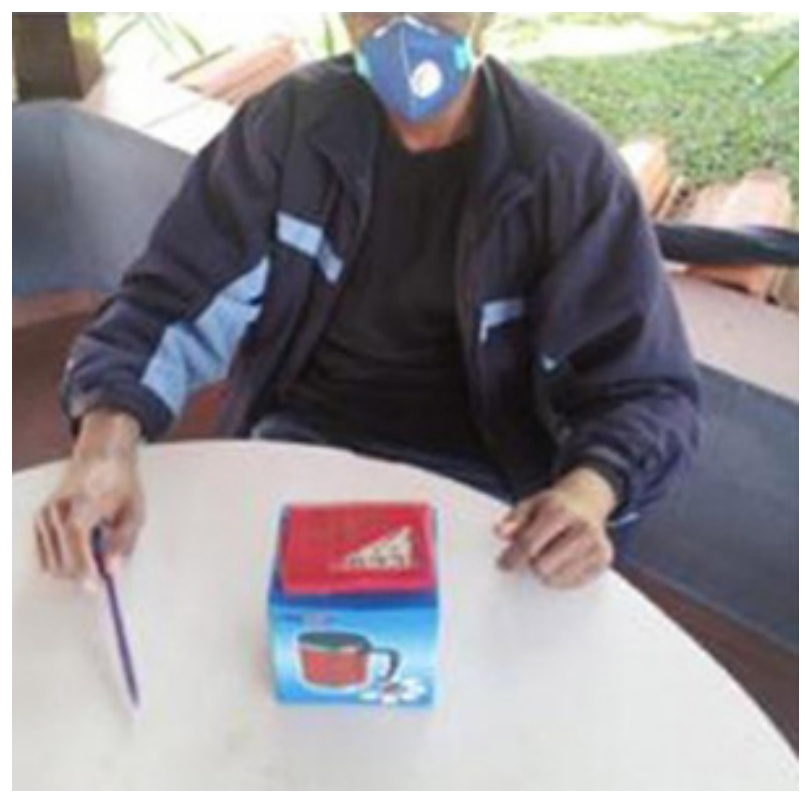

Figura 3 - Paciente com

brinde que ganhou no bingo

mensal do GATMO

Considerando as necessidades específicas de pacientes e acompanhantes, foram propostas tarefas que facilitassem a socialização, como sessões de exibição de filmes (seguidas por discussão), jogos de tabuleiro (nos quais todos pudessem jogar) e, principalmente, a realização de bingos, momento mais esperado do mês.

Gosto de tudo, mas o bingo é o melhor. A gente se reúne e se diverte, dá risada, esquece da vida e ainda ganha uns brindes. ( $\mathrm{P}, 54$ anos, masculino, diagnóstico de leucemia).

\section{CONSIDERAÇÕES FINAIS}

Na etapa pós-TMO, os pacientes vivenciam a ruptura do cotidiano, o rompimento de relações sociais, o estresse decorrente da doença prolongada, da hospitalização e do rigor da terapêutica instituída, além da perda e luto de projetos acalentados. Corre-se o risco da rotina ficar centrada somente nas atividades de cuidados com a saúde, com foco na preocupação com as questões da sobrevivência, cabendo à equipe multiprofissional o desafio de auxiliar o paciente a superar essa perspectiva que ameaça esmagar a subjetividade e estreitar os horizontes existenciais do transplantado [4].

Para Takatori [7], com a quebra do cotidiano, o processo de readaptação à nova realidade pode depender de uma estrutura que sustente esse período de transição e reorganização da vida, como é o caso dos grupos de atividades e oficinas terapêuticas. Os pacientes são considerados sobreviventes ao TMO, designação que remete a um tratamento considerado traumático e ameaçador à própria vida. Em determinado 
momento de suas vidas, esses indivíduos tiveram sua vida interrompida e podem apresentar por isso, dificuldades em reconstruir suas vidas e repovoar o cotidiano, o que leva à dificuldade de reinserção social.

As intervenções psicossociais implementadas no pós-TMO são destinadas a restituir a pessoa gravemente comprometida em sua autonomia a uma condição de atividade útil e construtiva [4]. Nesse contexto, as oficinas de atividades mostraram ser uma ferramenta de intervenção eficaz. Essas oficinas possibilitaram delinear um espaço/lugar de referência ao paciente e ao acompanhante, onde eles se sentem confortáveis para produzir, criar, expressar-se, desfrutar de um convívio em grupo, estar junto de pessoas que compartilham um desafio semelhante e que desejam fazer alguma atividade, através da qual possam se reconhecer com suas limitações e potencialidades. Experimentar um fazer junto e dar oportunidade ao paciente de se relacionar com pessoas diferentes podem ser consideradas medidas potencialmente terapêuticas. Os benefícios auferidos repercutem, em última instância, em uma melhor adesão ao tratamento, além de favorecer a reconstrução do cotidiano do transplantado.

Nesse sentido, tais grupos se configuram como um espaço de saúde, no qual os integrantes podem viver, a princípio, a relação terapeuta, paciente e atividades, crescentemente alcançando outras relações e espaços sociais, acreditando que a inserção social do sujeito acontece em um continuum que constitui - e se dá a experimentar - no cotidiano [7].

Frente ao reconhecimento da importância das oficinas terapêuticas do GATMO na fase pós-transplante, sugere-se que essa modalidade de intervenção terapêutica, ao ser implementada, tenha seu alcance testado desde o momento da espera pelo transplante (fase pré-TMO), no qual o paciente se encontra, muitas vez, sob o impacto do diagnóstico e da decisão de fazer o TMO, visando atenuar as dificuldades deste momento.

O projeto atual possibilitou ao aluno a oportunidade de conhecer a realidade do paciente transplantado de medula óssea, suas necessidades e potencialidades, participar de uma estratégia criativa de intervenção emocional e avaliar a efetividade desta. Acredita-se que essa seja uma oportunidade rica e fecunda para o graduando de aprendizagem de intervenções no contexto hospitalar.

\section{REFERÊNCIAS}

[1] CAMPOS, E. M. P.; et al.(2007). Intervenção em grupo: Experiência com mães de crianças com câncer. Psicologia em Estudo, v. 12, n. 3, p. 635-640, 2007.

[2] CONTEL, J. O. B.; et al. Aspectos psicológicos e psiquiátricos do transplante de medula óssea. Medicina Ribeirão Preto, v. 33, p. 294-311, 2000.

[3] MASTROPIETRO, A. P.; OlIVEIRA-CARDOSO, E. A.; SIMÕES, B. P.; VOLTARELLI, J. C.; SANTOS, M. A. et al. Relação entre renda, trabalho e qualidade de vida de pacientes submetidos ao transplante de medula óssea.

Revista Brasileira de Hematologia e Hemoterapia, v. 32, n. 2, p. 102-107, 2010.

[4] MASTROPIETRO, A. P.; et al. Vida ocupacional de pacientes sobreviventes 
ao transplante de medula óssea: estudo exploratório. Revista Brasileira de Orientação Profissional, v. 12, n. 2, p. 241-252, 2011.

[5] OLIVEIRA-CARDOSO, E. A.; et al. Qualidade de vida de sobreviventes do transplante de medula óssea (TMO): Um estudo prospectivo. Psicologia: Teoria e Pesquisa, v. 25, n. 4, p. 621-628, 2009.

[6] PONTES, L.; GUIRARDELlO, E. B.; CAMPOS, C. J. G. Demandas de atenção de um paciente na unidade de Transplante de Medula Óssea. Revista da Escola de Enfermagem USP, n. 41, v. 1, p. 154-160, 2005.

[7] AKATORI, M. A Terapia ocupacional no processo de reabilitação: Construção de cotidiano. O Mundo da Saúde. São Paulo, v. 25, n. 4, p. 371-383, 2001.

FLÁVIA ANDRÉA PRADO PATROCíNIo graduanda em Psicologia da Faculdade de Filosofia, Ciências e Letras de Ribeirão Preto da Universidade de São Paulo (FFCLRP-USP) e bolsista do Programa Aprender com Cultura e Extensão 2012-2013 - e-mail: prado_flavia@hotmail.com

ÉRIKA ARANTES DE OLIVEIRA-CARDOSO doutora em Psicologia e psicóloga do Departamento de Psicologia da Faculdade de Filosofia, Ciências e Letras de Ribeirão Preto da Universidade de São Paulo (FFCLRP-USP) - e-mail: erikaao@ffclrp.usp.br

BRUNA VIEIRA VON ZUBEN graduanda em Psicologia da Faculdade de Filosofia, Ciências e Letras de Ribeirão Preto da Universidade de São Paulo (FFCLRP-USP) e bolsista do Programa Aprender com Cultura e Extensão 2012-2013 - e-mail: brunavonzuben@hotmail.com

MANOEL ANTÔNIO DOS SANTOS professor associado da Faculdade de Filosofia, Ciências e Letras de Ribeirão Preto da Universidade de São Paulo (FFCLRP-USP) e coordenador do Laboratório de Ensino e Pesquisa em Psicologia da Saúde (LEPPS-USP-CNPq). Bolsista de Produtividade em Pesquisa do CNPq-e-mail: masantos@ffclrp.usp.br 